The Big Five Personality Traits, Big Two Metatraits and Social Media: A Meta-Analysis

\author{
Dong Liu $^{1}$ \\ Department of Psychology \\ Renmin University of China \\ bnuliudong@gmail.com \\ W. Keith Campbell ${ }^{*}$ \\ University of George \\ wkc@uga.edu \\ wkeithcampbell@gmail.com
}

${ }^{1}$ Correspondence concerning this article should be addressed to W. Keith Campbell, Department of

Psychology, University of Georgia, Athens, GA 30602-3013, fax 706.542.3275, E-mail: wkc@uga.edu, wkeithcampbell@gmail.com. Dong Liu and W.

Keith Campbell are co-first authors who contribute equally to this work. 


\begin{abstract}
This article reports a meta-analysis of the relationships between social network site use and the Big Five personality traits (openness, conscientiousness, extraversion, agreeableness, and neuroticism) as well as the Big Two metatraits (plasticity and stability). A random effect meta-analysis model was used to calculate the meta-results of Big Five. Extraversion and openness were the strongest predictors of SNS activities, while conscientiousness, neuroticism, and agreeableness only correlated with a few of the SNS activities. A meta-analytical structural equation model (SEM) further demonstrated that plasticity was positively correlated with SNS activities, whereas stability was a negative predictor. Practical implications for social media industry and users are discussed.
\end{abstract}

Keywords: Big Five, Social Network Site (SNS), Big Two, meta-analysis, personality, Five Factor Model 


\section{The Big Five Personality Traits, Big Two Metatraits and Social Network Site Use: A Meta-Analysis}

The practice of checking profiles and posting updates on one or more social network site (SNS) has become part of everyday

life for many Americans. On SNSs, individuals can broadcast large amount of personal information (which can be in many forms such as text, images, links, or video) and make social requests to broad audiences. They can also comment directly on the posts friends placed on SNSs or reply through other online channels (e.g., Twitter, Snapchat) or even alternate channels offline (e.g., phone calls, text messages or face to face). But what drives these SNS behaviors? Examining the role of personality traits in predicting SNS activity is one potentially useful way to understand the psychology of SNS use. As such, a burgeoning literature has emerged examining personality and SNS activity. One major focus has been on examining the Big Five or Five Factor Model (FFM) of personality traits and SNS activity, with the specific traits being extraversion, openness to experience, agreeableness, conscientiousness and neuroticism.

Although considerable evidence (reviewed below) suggests that certain Big Five personality traits are associated with certain aspects of SNS use, several issues connecting personality traits and SNS use remain ambiguous. First, after one decade of investigating the relationships between the Big Five traits and SNS use, there are no clear estimates regarding the extent to which specific personality traits are linked to SNS behaviors. Second, and related, prior studies remain limited either in the sample size or in the scope of social networking online (e.g., focusing solely on global SNS usage or a single SNS). There is not an integrated picture of 
how personality is linked the digital footprint people leave on SNS, not only in terms of time spent on SNS, but also in terms of particular online activities. Third, while there has been a focus on the Big Five at the trait level, there has been no research on the link between SNS use and the two higher order factors of the Big Five, or Big Two metatraits (e.g., DeYoung, 2006; Digman, 1997).

Theoretically, an opportunity exists to pair a Big Two model of personality with a cybernetic approach in order to provide a plausible higher level account of personality and SNS use (DeYoung, 2015). Given these questions, the time is ripe for a systematic review and analysis of the online social networking literature. This review thus has three goals:

1. Evaluate the connection between Big Five personality traits and global SNS use, and test their robustness against methodological artifacts.

2. Evaluate the connection between Big Five personality traits and specific SNS activities, and test their robustness against methodological artifacts.

3. Evaluate the connection between Big Two metatraits and various SNS activities, and test their robustness against methodological artifacts.

We address these goals with a series of meta-analyses but first provide a more detailed discussion of the theory and recent findings on personality and social networking online.

\section{Aspects of Social Network Site Use}


Before articulating our hypotheses, it is useful to appreciate the main quantifiable aspects of SNS usage studied in the literature. These have been measured in research studies and hence form the main factors in our meta-analysis. That is, they are what we sought to predict based on personality.

The first is simply the aggregate total amount of activity on SNS. This represents the person's overall use of SNSs. It could be measured with total time spent on such sites, frequency of visiting them, or intensity of activity.

Second, status updates refer to verbal disclosures about oneself. Essentially one posts information to make it available to others to see. This goes strongly to the self-presentational component of SNS use.

Third, interactions refer to responses specifically to other people's disclosures or comments. The person thus replies to or comments on something another person has communicated via the SNS. Interactions engage the individual with a specific other person. The simplest interaction is to click the "Like" response. Interactions leave visible traces, unlike merely browsing or viewing someone's page.

Fourth, SNSs enable people to post photos, which may be of themselves or of personally meaningful or interesting scenes. The number of photos on an individual's site can therefore be counted.

Fifth, and finally, Facebook enables users to form ostensible or virtual social bonds by mutually declaring each other to be "friends." Although a large proportion of SNS friends come from offline, many of them are only slight acquaintances, in some cases 
having met once or even not at all (Liu, \& Yang, 2016). Each person's tally of such friends is kept by the SNS, thereby enabling researchers to explore the size of these friendship networks.

\section{A Cybernetic Approach to Personality}

In the present study, we focus primarily on personality traits as part of a cybernetic system (DeYoung, 2015). This cybernetic approach links personality traits to specific self-regulatory actions. For example, the Big Five trait of extraversion is, according to a cybernetic model, primarily linked to environmental rewards, approach orientation and behavioral exploration (as opposed to the cognitive exploration seen with openness to experience; DeYoung, 2014).

This cybernetic approach to personality can also be used to understand the higher order metatraits, or Big Two, of plasticity (extraversion + openness) and stability (low neuroticism + conscientiousness + agreeableness). The cybernetic approach can even be used to understand the ten lower order personality aspects of the Big 5, although these will not be focused on in this research.

The cybernetic approach is useful for understanding the link between personality traits and metatraits in SNS use because the focus is on action - for example, these networks are actively built and used by individuals so thinking about personality as goal driven and self-regulatory will potentially aid in understanding SNS. Other theoretical approaches to the Big Five including Big Five as reputation (Hogan, 1996), Big Five as universal and biologically based structures (McCrae \& Costa 1997) and Big Five as lexical descriptions (John \& Srivastava, 1999) do not seem as directly useful when thinking about personality and SNS activity. 


\section{The Big Five Traits and Social Network Sites Use}

The Big Five is the most widely used and well-validated taxonomy of personality traits, using five dimensions to describe personality: extraversion, neuroticism, agreeableness, conscientiousness, and openness to experience/intellect (for a recent review, see John, Naumann, \& Soto 2008). These Big Five traits can capture a very large amount of variance in personality, hence the moniker of "Big".

Extraversion is as associated with activity, social gregariousness, optimism, drive, and talkativeness (e.g., Costa \& McCrae, 1992) as well as excitement-seeking (H. J. Eysenck \& Eysenck, 1975) and seeking social attention (Ashton, Lee, \& Paunonen, 1999). In cybernetic terms, as noted, extraversion is associated with reward seeking and behavioral exploration. It is likely that extraverts will engage in higher amounts of social activity on SNS because they are reward seeking and sociable. Prior studies on SNS have consistently found that extraverts not only spend more time on SNS (Wilson, Fornasier, and White, 2010), had more Facebook friends (Ong et al., 2010; Ross et al., 2009), and also are central in many social networks (Feiler \& Kleinbaum, 2015).

Conscientiousness can be described as the tendency to follow rules, be industrious and dutiful, and resist immediate gratification in the interest of longer term goals. Indeed, from a cybernetic perspective, conscientiousness is primarily about protecting long term goals from short term temptations (DeYoung, 2015). When framed in this way, it is reasonable that conscientiousness would be associated with lower levels of SNS - assuming that SNS is often tempting distractions that interfere with longer term goals. This 
has been found in the research: individuals scoring high on conscientiousness were more likely to spend less time on SNS while completing other tasks. (e.g., Carrier, Cheever, Rosen, Benitez, \& Chang, 2009). Similarly, Moore and McElroy (2012) found that conscientiousness was associated with greater regret over inappropriate Facebook posts, suggesting that conscientious individuals present themselves cautiously online and that conscientiousness might even be negatively associated with SNS use.

High scores on neuroticism are associated with anxiety, depressive affect, and emotional instability (Costa \& McCrae, 1992). In cybernetic terms, neuroticism is "defensive response" to threat (DeYoung, 2015). It is plausible that in many cases SNS behavior can be used to mitigate neuroticism by provide an outlet for conversation or social connection. Supporting this hypothesis, research has shown that neurotic individuals are more likely to blog (Guadagno et al., 2008) and post on their walls (Ross et al., 2009; Moore \& McElroy, 2012).

Openness to experience includes the tendency and ability to seek, detect, comprehend, and utilize patterns abstract and sensory of information. High openness is also associate with an interest in art and fantasy (Costa \& McCrae, 1992). High openness to experience is reflected in curiosity and novelty-seeking; low levels are evident in preferences for adhering to convention and established patterns of behavior (John \& Srivastava, 1999). In cybernetic terms, openness is associated with exploration and curiosity (DeYoung, 2015). These psychological states seem to be closely related to new media use. It is expected that those who scores high in openness are more likely to try all SNS activities. In previous studies, openness has emerged as a reliable predictor of SNS use (Moore, 
\& McElroy, 2012).

Agreeableness reflects one's interpersonal orientation towards others, including sympathy, courteousness, interpersonal flexibility, kindness, trust and forgiveness (Costa \& McCrae, 1992). In cybernetic terms, agreeableness is associated with cooperation and relating positively to others (DeYoung, 2015). Individuals who score high on Agreeableness are arguably more likely to be involved with online interaction and to engage in more SNS activities with offline friends.

\section{The Big Two Metatraits}

The Big Five traits can be captured at higher orders by metatraits. Our focus is on the Big Two metatraits (DeYoung, 2006; Digman, 1997). These Big Two have several names and associated conceptualizations. One is the metatrait more closely associated with extraversion, dominance, and openness, termed plasticity (DeYoung, 2015), beta (Digman, 1997), agency (Bakan, 1966), status (Wiggins, 1979), and dominant (Leary, 1957). The other is the metatrait more closely associated with agreeableness, warmth, and peace, termed stability (DeYoung, 2015), alpha (Digman, 1997), communion (Bakan, 1966), and love (Leary, 1957; Wiggins, 1979).

These models of the Big Two vary in specificity, so scales such as those developed by Wiggins primarily capture extraversion and agreeableness (McCrae \& Costa, 1989) whereas the explicit efforts to capture metatraits such as those by DeYoung and Digman capture more of the Big Five, with plasticity/beta primarily extraversion and openness and stability/alpha primary agreeableness, (low) neuroticism and conscientiousness. 
There are two major advantages of using the Big Two to meta-analytically model the SNS literature. First, there is the advantage of theoretical simplification. If SNS activity can be reasonably captured by the action of two traits we can generate a very parsimonious theoretical account of the personality-SNS link. Second, there is the empirical issue of handling multiple comparisons. Looking at the Big Five traits with 5 outcomes yields 25 tests; with the Big Two that number is cut by $60 \%$ to ten tests. Both cases will likely yield "noise" - even in meta-analyses - but there will be less with the Big Two. In addition to these theoretical and empirical reasons, no research has actually examined the Big Two and SNS activity; doing so will add a new finding to the literature.

\section{The Big Two Metatraits and Social Media from a Cybernetic Perspective}

In DeYoung's cybernetic model (2015), personality is considered a goal directed cybernetic system, of which plasticity and stability are the two metatraits. In the cybernetic system, these two metatraits respectively fulfill two basic needs of any complex self-regulating organism existing in the unpredictable environment: plasticity is associated with exploration and goal creation; and stability with goal maintenance in the face of threat and distraction (DeYoung, 2015). To expand: the metatrait plasticity, defined as the combined variance of extraversion and openness/intellect, appears to reflect an exploratory tendency and ability to actively engage with the possibilities of the environment, both generating and attending to novel aspects of experience (DeYoung, 2015). Plasticity can be described as the degree to which the personality system is prone to generating new goals, new interpretations of the present state, and new strategies to pursue existing goals. People high in plasticity are not only prone to respond to anomalies more flexibly and 
eagerly when they appear unexpectedly, they also tend to seek out the unknown voluntarily. Plasticity should also be associated with dopamine because of the link to approach and reward. In terms of actual behavior, plasticity is associated with interpersonal connection, parties, jokes, and travel (see Hirsh, DeYoung and Peterson, 2009, Table 3 for fuller account). Stability, in contrast, reflects the capacity of the cybernetic system to resist disruption. Following an encounter with an anomaly, people high in stability will resist replacing their operative goal with immediate goals (like expressing anger or pursuing a distraction) that interfere with longer-term goals. In contrast, the characteristic adaptations of people low in stability are frequently interrupted by emotions, impulses, and doubts. Stability should also be associated with serotonin because of the link to (low) anxiety and calm. In terms of actual behavior, stability is associated with resisting impulsive behaviors - it is associated with less anger and nervousness, less joking, less overeating and less sex (Hirsh et al., 2009).

In terms of SNS use, plasticity should predict greater social network site use while stability should predict less. Think of a foray into a specific social network, say Facebook or Snapchat. Plasticity should theoretically drive trying new procedures in the system, building links, and creating new material (e.g., posting selfies); once these new structures are created, stability should work to maintain them. So, with a site like Facebook, someone's plasticity score should predict actively engaging with new friends or groups; whereas someone's high stability score should predict maintaining those new relationships. The challenge, of course, is that often SNS use is seen as a distraction from work or "real life." Because of this, stability should actually predict less SNS use because the larger 
goal of successful work or family is being protected from the distraction of SNS. This might be easier to see in the reverse: Social media can be a massive distractor so individuals low in stability are more likely to be tempted by it.

\section{The Present Research}

The present meta-analysis focuses on the associate between the Big Five and Big Two personality models and SNS activity. Our theoretical approach is based on a cybernetic model of personality traits that considers the importance of traits as part of a self-regulatory system. The Big Five traits will be collected and tested in a standard meta-analytic fashion. The Big Two will be assessed by conducting a SEM meta-analysis of Big Five personality traits. Overall, we predict that plasticity will be positively related with the spectrum of SNS activities, and stability will be negatively related.

\section{Method}

\section{Literature Search}

Four methods were used to search for relevant studies. First, we retrieved articles through a detailed search of PsycINFO, PsycArticles, EBSCO_ERIC, Medline, Communication and Mass Media Complete (CMMC), Google Scholar, and ProQuest Dissertations \& Theses. The following key words were used in combination: personality, Big Five, Five Factor Model, FFM, Big5,

Agreeableness, Conscientiousness, Extraversion, Openness to Experience, and Neuroticism, Facebook, Myspace, Social Network Sites, SNS, and social media. Second, we conducted systematic searches using Chinese, Japanese and French translation of the key words in 
Google Scholar, CNKI and CiNii. Third, we searched the in-press or online-first articles. Finally, we searched the conference database: International Communication Association Conference (ACM), International Conference on Web and Social Media (ICWSM), Annual SIGCHI Conference, ACM digital library. We contacted authors of relevant posters or presentations at these conferences to ask for information about their studies. We did not place any limitations on age, geography, SNS types, or other sample characteristics in our search. The literature search encompassed articles published up to March 01, 2016.

\section{Inclusion and Exclusion Criteria}

Our search resulted in 5,062 records for screening to identify eligible studies. After examining the titles and abstracts of all the references and discarding obviously irrelevant ones, 136 articles were identified as highly relevant. 90 articles were excluded from our analysis based on the following criteria: (a) the studies needed to be empirical and quantitative (i.e., review, theoretical, and qualitative studies were excluded); (b) personality was measured by scales with promising reliability ( $\alpha>$.70); (c) global measures of social network sites use (e.g., intensity, time, or visiting frequency) were provided; (d) pearson correlation coefficients were provided; otherwise, sufficient information from which an effect size could be derived needed to be available; (e) studies examining SNS user and non-user differences on personality were excluded, even if they provided t-statistics; (f) scales that blended elements of two or more Big Five categories were excluded; (g) studies violating the assumption of independent samples were excluded. If a study utilized the same data as any previously coded studies, it was considered a violation of independent samples. In the cases where the 
results of a specific data set were re-used or otherwise duplicated in more than one publication, we chose the publication that contained most information or was published in the journal with the highest impact factor. Finally, we obtained 38 studies, see Appendix. This final set of studies was selected in advance of the analyses. That means there was no opportunity for (inadvertent) bias in study selection to creep in due to observation of results.

\section{Coding}

Studies that met the inclusion criteria were coded for sample characteristics (country, proportion of female, average age, and sample size) and information needed for the publication bias test (i.e., publication status and publication year). The first author developed a coding manual that specified the coding categories and possible codes to be used for each study. Following the coding manual, the first author and a doctoral student coded all information contained in the 38 studies. Ninety-five percent of the two coders' coding reached an agreement. All disagreements were resolved through discussion among the coders.

\section{Multiple Dependent Results from a Single Study}

When a study included multiple independent effect sizes (e.g., correlation coefficients from separate independent samples), each effect size was coded separately. When a study reported multiple dependent effect sizes, however, simultaneously including them in a single meta-analysis can pose calculation problems of lower error variance estimate and inflating significance tests (Cooper, Hedges, \& Valentine, 2009). To isolate independent findings for use in each meta-analysis, the following procedures were conducted: 
(a) we conducted separate meta-analyses for different personality traits. Hence, only if a study reported multiple results for a single type of association would we need to address independence of effect sizes further; (b) if a study reported one correlation for multiple times, we aggregated them into a single effect with within-study aggregation functions from $M A c$ package in $R$ (Del Re, \& Hoyt, 2010).

\section{Computation of Effect Sizes}

Firstly, correlation coefficients $(r)$ for the relationships between SNS usage and personality traits were recorded. Then, we used the meta-analytic procedures of Hunter and Schmidt (2004) to correct observed correlations for measurement error in both the predictor and the criterion scores. Correlations were corrected individually with internal reliability. The large majority of studies reported the reliability needed for the correction. For the few cases in which reliability was not provided, we used the average value across other studies to complete the correction. The correction work was completed with the $M A c$ package from $R$ (Del Re $\&$ Hoyt, 2010). Finally, the meta-analyses were conducted using effect sizes that were transformed to Fisher's Z, and using study weights with $\omega=$-3 (Lipsey \& Wilson, 2001). Effect sizes were then transformed back into correlations when reporting the results of the analyses for ease of interpretation.

The homogeneity of the effect sizes was assessed using a $Q$ statistic (Borenstein et al., 2009) and $I^{2}$. A significant $Q$ provides evidence that true effects vary across studies and warrants a random model. But the $Q$-test may be oversensitive when there are many 
studies/effect sizes (especially if the $N$ 's are high). When the $Q$ statistic was significant, we further checked $I^{2}$, which provides information about the magnitude of heterogeneity (Huedo-Medina, Sánchez-Meca, Marín-Martínez, \& Botella, 2006), to decide whether random-effects or fixed-effects model should be employed. All analyses were completed in Comprehensive Meta-Analysis version 3 (Borenstein et al., 2005).

\section{SEM Meta-Analysis}

Following Viswesvaran \& Ones (1995), we used a meta-analytic correlation matrix as the primary data for testing our models. The correlations in this matrix were from the overall effect size for each cell. We ran our mediation model in Mplus 7, testing the significance of the link between meta-traits and SNS use behavior (see figure 1). The sample size employed was the harmonic mean across all studies included in the correlation matrix. Model fit was assessed using goodness-of-fit indices such as the comparative fit index (CFI), the root mean square error of estimation (RMSEA), and the standardized root mean squared residual (SRMR). Based on the recommendations of $\mathrm{Hu}$ and Bentler (1999), CFI values exceeding .95 indicates good model fit, whereas RMSEA and SRMR should not surpass values of .08 and .06 , respectively.

\section{Publication Bias Analysis}


Following the standard practice for testing publication bias in meta-analysis, we applied three methods: (a) P-Curve analyses (Simonsohn, Nelson, \& Simmons, 2014); (b) examining funnel plots of effect size standard errors to check possible bias. All data were analyzed together for publication bias.

\section{Results}

Substantial aggregated sample sizes were obtained for correlations with each Big Five dimension (from 6,421 for correlations with conscientiousness to 10,223 for correlations with extraversion). The sample sizes of the studies included in the meta-analyses ranges from 39 to 1,452 . The mean was 306. Mean age of the sample in these studies was 26.37, ranging from 16.98 to 46 years. Mean proportion of female in the sample was .61 , ranging from .17 to .87 . No outlying effect sizes were identified. The data came from 11 countries (or regions). Please check Appendix for details of the included studies.

This meta-analysis provides a high level of power for statistically predicting personality traits' association with social networking online. After the statistics were coded, meta-analyses were conducted with all correlations for each Big Five dimension and global SNS use. In this analysis, population values for $r$ and were estimated with the Hunter and Schmidt (2004) random effects method. $Q$ value was calculated on the basis of correlations that were corrected for internal reliability (alpha). Although Cochran's $Q$ is commonly reported, $I^{2}$ (Higgins \& Thompson, 2002) has been recommended as a supplement to $Q$ when one assesses heterogeneity because, unlike $Q, I^{2}$ allows researchers to quantify heterogeneity and to compare the degree of heterogeneity within different analyses. 
Thus an $I^{2}$ value of $75 \%$ indicates that three quarter of the variation between studies reflects systematic or heterogeneous variation,

rather than random sampling error.

\section{SNS Use and Big Five Traits}

The results demonstrated that only extraversion $(r=.11, p<.001)$ and neuroticism $(r=.10, p<.01)$ were significantly associated with global SNS use, see Table 1.

Further meta-analyses delineated a more detailed picture between FFM traits and SNS use activities, see Table 2 and Figure 2. As expected, extraversion had the most links to SNS activities. Extraversion was positively related to more than half of the SNS activities, with the largest link to total SNS friends $(r=.34, p<.001)$, but also a link to photos $(r=.18, p<.001)$ and interactions $(r$ $=.14, p<.001)$.

Openness was also positively linked to the majority of SNS activities examined. It was positively link to SNSs games $(r=.28$, $p<.001)$. It also had a connection to information seeking $(r=.19, p<.01)$, photos posting $(r=.14, p<.01)$ and status update $(r=.12$, $p<.001)$.

Agreeableness was positively related to photo posting $(r=.18, p<.01)$, but negatively correlated with SNS games $(r=-.05, p$ $<.01)$. Conscientiousness was the only personality traits negatively related or unrelated to all SNS activities measured. It was 
negatively correlated with SNS gaming $(r=-.22, p<.05)$ and information seeking $(r=-.12, p<.001)$. Neuroticism was only associated with SNS status update $(r=.13, p<.05)$.

Our second goal was to see how much variance the Big Five explain together in each of these SNS outcomes by estimating inter-correlations from a meta-analysis and then combing them. The Big Five was not very effective for predicting global SNS use, with $R^{2}=.03$. But the variances of SNS activities explained by Big Five were much larger, ranging from .05 to .18, see Table 3 .

The hierarchical multiple regression analyses were presented in Table 3, where we examine the SNS activities explained by the Big Five Factor. Standardized regression coefficients and $R^{2}$ were estimated using meta-analytic FFM inter-correlations reported in Linden, Nijenhuis, and Bakker (2010; see Table 2 in their study), combined with FFM traits to SNSs activities correlations estimated in this study. As expected, the FFM traits together did explain a significant portion of variance in SNS activities, ranging from .03 to .18. The variance explained by FFM for global SNS use was the lowest $\left(R^{2}=.03\right)$ and gaming the highest $\left(R^{2}=.18\right)$ with total friends a close second $\left(R^{2}=.16\right)$.

\section{SNS Use and Big Two Traits}

Meta-analytic structural equation modeling was used to test our proposed Big Two model. We first constructed the meta-analytic correlation matrix with FFM inter-correlations reported in Linden, Nijenhuis, and Bakker (2010). We then entered that meta-analytic correlation matrix into a structural equation modeling analysis using Mplus 7 (Muthén, \& Muthén, 2012). Given that the 
sample sizes differed across the various cells of the matrix, we used the harmonic mean sample size to compute standard errors

(Viswesvaran \& Ones, 1995). As less weight is assigned to large samples, use of the harmonic mean results more conservative

estimates. The statistics in Table 4 display the results for the relationship between SNS activities and Big Two personality metatraits:

plasticity positively predicted all SNS activities, whereas stability negatively predicted all SNS activities except for SNS interaction.

With confirmatory factor analyses (using $M L$ estimates) we tested the proposed models. Big Five loaded as expected on the two metatraits Plasticity $(\mathrm{O}, \mathrm{E})$ and Stability $(\mathrm{C}, \mathrm{A}, \mathrm{N})$. Nearly all the proposed models had a good fit to the data (see the $\chi^{2}, R M S E A$, CFI, and SRMR value in Table 4). Adding new correlation paths between Big Five personalities (based upon medication indices and significance results), the resulting model provided acceptable fit to the data, $\chi^{2}$ ranging from 94.45 to $711.17, R M S E A$ from .06 to .14 , CFI from .88 to .96, SRMR from .026 to .080 . However, the model fit for the Big Two personality metatraits predicting SNS gaming $(C F I=.881, S R M R=.080, R M S E A=.144)$ was poor. We tried to modify this model by adding correlation paths between the Big Five personality traits, but the model fit still remained poor.

The variance explained by the Big Two model ranged from .036 to .169. Specifically, the Big Two explained the most variance for total SNS friends, and the least variance for the SNS interaction. The standardized path from Stability and Plasticity were reported in Table 4. The standardized path coefficient from Plasticity to SNS activities ranged from .227 for global SNS use to .557 for photo posting on SNS. The standardized path coefficient from Stability to SNSs activities ranged from -0.015 for SNS interaction to -.36 for 
SNS information searching. Overall, plasticity explained more SNS activities variance than stability: individuals with high levels of plasticity tend to spend more time on SNS and more actively participate into SNS activities, and individuals with high levels of stability tend to spend less time on SNS and less actively participate into SNS activities.

\section{Moderators of the Personality-SNS Global Use Relationship}

Demographic variables-gender, age and culture (Eastern vs Western) -were used as possible moderators of the relationship between personality and SNS global use. Here average gender and age of the sample was used as continuous predictors, while culture was treated as categorical. Guided by Suh, Diener, Oishi, and Triandis' (1998) coding, we coded studies conducted in Korea and Taiwan as the Eastern collectivist culture, and the rest countries in Europe and North America the Western individualistic culture. The results showed there was a tendency for conscientiousness to correlate less strongly with SNS global use for females $(B=-.608$, S.E.

$\left.=.173, p<.001, R^{2}=.54\right)$ and Eastern culture $\left(\rho_{\text {east }}=-.085, p>.05,95 \% C I[-.227, .061] ; \rho_{\text {west }}=.064, p>.05,95 \% C I[-.019, .147]\right.$, $\left.Q_{\text {between }}=3.042, p=.08\right)$.

\section{Publication Bias Analysis}

We checked the funnel plots, and non-symmetrical patterns were found in the funnel plot of extraversion and general SNS use (see figure 3). Six studies were imputed on the left side of the estimated population effect size. The corrected effect size by including these 6 imputed effect size was .07, with $95 \%$ C.I. from .03 to .12. 
P-hacking, or the selective publication of statistically significant results while suppressing null findings, is a significant problem in contemporary social psychology. In light of this fact, we conducted P-curve analyses (Simonsohn, Nelson, \& Simmons, 2014) to confirm that the above findings have evidential value and are not a result of p-hacking or publication bias. These analyses were conducted with applications from www.p-curve.com. All p-curves showed a shape that is right skewed and not flatter than $33 \%$, suggesting that the data for all meta-analysis of general SNS use and have evidential value and that p-hacking is unlikely to have occurred (see figure 4). These results showed that the non-significant effect size of agreeableness, consciousness, and openness may be underestimated.

\section{Discussion}

This research set out to answer three primary questions: How are the Big Five related to general SNS use? How are the Big Five related to more specific SNS activities? And how can the Big Two explain these patterns of SNS use?

In terms of global SNS use, there was a rather small link with the Big Five. Only extraversion and neuroticism showed significant (and small) correlations. The associations with personality became more apparent when specific SNS activities like photo posting, linking with friends, status updates, etc. were examined. In these analyses about a third of the relationships were significant with most of the action involving extraversion and openness. Of these two traits, extraversion was the best predictor of SNS activities in terms of association strength and the number of SNS activities it significantly correlated with; however, the relationship seemed to 
be largely accounted for by the variance extraversion shared with other personality factors. Controlling for correlations between personality factors in regression analysis resulted in a significant drop in the strength of the association.

The three remaining factors - neuroticism, conscientiousness and agreeableness — were less consistently associated with SNS activities. Moreover, there were some inconsistencies between zero-order correlation and regression results. For example, although neuroticism was only positively associated with neuroticism in zero-order correlation, we found its association with all other SNS activities significant. This inconsistency may be the result of some form of suppression effect (Paulhus, Robins, Trzesniewski, \& Tracy, 2004).

On average, the links between personality and SNS are relatively small but range from non-existent to large, as in the case of extraversion and number of SNS friends (Gignac \& Szodorai, 2016).

Plasticity and stability — the Big Two personality metatraits (DeYoung, 2006) — had clear and opposite patterns of associations with SNS use and activities. Plasticity was positively correlated with SNS activities in general; whereas stability was t negatively associated. In comparison to the findings with the Big Five, the Big Two showed a relatively consistent pattern of results with most relationships significant.

\section{Implications}

The most obvious implication is that SNS use is linked to personality in meaningful ways, but that these links are modest in 
size. This suggests that personality can be an important piece in solving the puzzle of SNS behavior, but more many more pieces are needed. Furthermore, the largest effect sizes associated with personality come from matching specific activities (e.g., friending,

gaming) and specific Big Five traits (e.g., extraversion, openness). This suggests that more targeted traits (e.g., personality facets) and more specific behaviors (e.g., specific types of games) might yield even stronger effects. This will be an interesting question for future research.

Theoretically, this meta-analysis supports the utility of using the Big Two metatraits to broadly conceptualize personality and SNS use and activity. Unlike freeways or road systems, SNSs are not built out before use. Instead, SNS growth emerges from users themselves building connections and content. This meta-analysis shows that these SNS builders and creators are largely high in the metatraits of plasticity, or extraversion and openness at the trait level. The importance of plasticity suggests that SNSs should not be thought of as static networks but rather constantly emerging, dynamic spaces. Given that, SNSs will likely keep growing if they continue matching goals appropriate from high plasticity individuals, which from a cybernetic perspective would include opportunities for reward seeking, excitement, creativity and novelty. On the other hand, it also suggests that SNS shall constantly roll out new functions and interface to satisfy the ever-changing appetite of its highly plastic consumers.

In our meta-analysis, this Big Two metatrait of stability also predicted SNS activities but in a negative way. From a cybernetic perspective, stability is associated with refraining from a variety of behaviors associated with disruptive impulse (DeYoung, 2015). 
Tweeting, updating your status on Facebook or checking LinkedIn when you are working can be seen as disruptive behaviors that distracts your attention from your current tasks. For example, Judd (2014) found that Facebook was one of the most common computer-based activities among Australian students and Facebook use included significantly more multitasking behaviors and less focused behaviors. Our finding showed that people of low stability may be frequently distracted by social media use from work or study, whereas those high in stability are more likely to hold back the temptation of SNSs, as they have higher self-control.

From a SNS building perspective, the challenge with the high plasticity/low stability traits associated with use is that these networks will be constantly in flux and somewhat lacking in stability. Stability is, of course, important for durability of a system.

Theoretically, a solution to this problem would be to make SNS activities consistent with long term goals so that individuals higher in stability would use them more and thus help maintain (if not grow) the networks. This could be done by linking certain SNS activities with work outcomes, for example framing "networking" as an important business or professional practice. More broadly,

organizations that could positively link organizational goals with individual goals and SNS activity will find a more positive link with metatrait level stability.

\section{Limitations and Future Directions}

Although the metatrait level of personality structure appears highly effective at capturing the relations between personality and SNS activities, future research might examine whether any correlations with SNS activities are particularly strong at a level of 
personality structure lower and more specific than the Big Five, such as the 10 facet-level traits suggested by the cybernetic approach (DeYoung, 2015). Moreover, the present study was merely correlational, and causal inference are speculative. We have followed the standard procedure of assuming that personality traits are predisposing causes and behavior is the result, but the opposite direction cannot be ruled out. For example, it could be the case that people who use more SNS become less stable and more plastic. And, of course, there could be bidirectional effects or feedback loops involved. In addition, we have argued that a cybernetic approach is useful for understanding personality and SNS use, but this needs to be tested more specifically. There are many studies that could be done looking at goals, contexts and even brain activity to better understand personality and social media. Finally, in certain cases we have less than five studies between particular personality traits and specific SNS activities, so the generalizability of these small meta-analyses are limited. More future work is needed to illuminate the relationship between different aspects of SNS activities and personality.

\section{Conclusion}

Social networking sites are linked to the Big Five personality traits and Big Two metatraits in meaningful ways. From a Big Five perspective, most associations involve extraversion and openness; from a Big Two perspective, high plasticity and to a lesser extent low stability are the main predictor of SNS use. These results provide interesting insights into the nature of social networking but more research is needed. In particular, theoretical and empirical work on the Big Two and social media would be useful. 


\section{References}

References marked with an asterisk indicate studies that are included in the meta-analysis.

*Bai, S., Gao, R., \& Zhu, T. (2012). Determining personality traits from Renren status usage behavior. In Computational Visual Media (pp. 226-233). Springer Berlin Heidelberg.

Bakan, D. (1966). The duality of human existence: An essay on psychology and religion. Oxford: Rand McNally. $\underline{\text { http://dx.doi.org/10.1086/224499 }}$

*Chen, J. V., Widjaja, A. E., \& Yen, D. C. (2015). Need for affiliation, need for popularity, self-esteem, and the moderating effect of big five personality traits affecting individuals' self-disclosure on Facebook. International Journal of Human-Computer Interaction, 31, 815-831. http://dx.doi.org/10.1080/10447318.2015.1067479

*Chorley, M. J., Whitaker, R. M., \& Allen, S. M. (2015). Personality and location-based social networks. Computers in Human Behavior, 46, 45-56. http://dx.doi.org/10.1016/j.chb.2014.12.038

*Chou, H. W., Chang, K. C., \& Lin, Y. H. (2012). Facebook and Google Usage in Taiwan's College Students. In Eleventh Wuhan International Conference on e-Business. Paper 91. http://aisel.aisnet.org/cgi/viewcontent.cgi? article=1009\&context=whiceb2011

*Correa, T., Hinsley, A. W., \& De Zuniga, H. G. (2010). Who interacts on the Web? The intersection of users' personality and social 
media use. Computers in Human Behavior, 26, 247-253. http://dx.doi.org/10.1016/j.chb.2009.09.003

*De Cock, R., Vangeel, J., Klein, A., Minotte, P., Rosas, O., \& Meerkerk, G. J. (2014). Compulsive use of social networking sites in Belgium: prevalence, profile, and the role of attitude toward work and school. Cyberpsychology, Behavior, and Social Networking, 17, 166-171. http://dx.doi.org/10.1089/cyber.2013.0029

DeYoung, C. G. (2006). Higher-order factors of the Big Five in a multi-informant sample. Journal of Personality and Social Psychology, 91, 1138-1151. http://dx.doi.org/10.1037/e511092014-241

DeYoung, C. G. (2015). Cybernetic big five theory. Journal of Research in Personality, 56, 33-58. http://dx.doi.org/10.1016/j.jrp.2014.07.004

Digman, J. M. (1997). Higher-order factors of the Big Five. Journal of Personality and Social Psychology, 73, $1246-1256$. http://dx.doi.org/10.1037/0022-3514.73.6.1246

Feiler, D. C., \& Kleinbaum, A. M. (2015). Popularity, similarity, and the network extraversion bias. Psychological Science, 26, 593-603. http://dx.doi.org/10.1177/0956797615569580

Gignac, G. E., \& Szodorai, E. T. (2016). Effect size guidelines for individual differences researchers. Personality and Individual Differences, 102, 74-78. http://dx.doi.org/10.1016/j.paid.2016.06.069

*Giota, K. G., \& Kleftaras, G. (2013). The role of personality and depression in problematic use of social networking sites in Greece. 
Cyberpsychology: Journal of Psychosocial Research on Cyberspace, 7(3), article 1. http://dx.doi.org/10.5817/cp2013-3-6

*Glass, R., Prichard, J., Lafortune, A., \& Schwab, N. (2013). The influence of personality and Facebook use on student academic performance. Issues in Information Systems, 14, 119-126.

*Golbeck, J., Robles, C., \& Turner, K. (2011). Predicting personality with social media. In CHI'11 Extended Abstracts on Human Factors in Computing Systems (pp. 253-262). ACM. New York, NY.

*Gosling, S. D., Augustine, A. A., Vazire, S., Holtzman, N., \& Gaddis, S. (2011). Manifestations of personality in online social networks: Self-reported Facebook-related behaviors and observable profile information. Cyberpsychology, Behavior, and Social Networking, 14, 483-488. http://dx.doi.org/10.1089/cyber.2010.0087

*Halevi, T., Lewis, J., \& Memon, N. (2013). Phishing, Personality Traits and Facebook. ArXiv preprint arXiv: 1301.7643.

Hamid, N. A., Ishak, M. S., \& Yazam, S. S. N. M. (2015). Facebook, YouTube and Instagram: Exploring Their Effects on Undergraduate Students' Personality Traits. The Journal of Social Media in Society, 4, 138-165.

Hogan, R. (1996). A socio-analytic perspective of the Five Factor Model. In J. S. Wiggins (Ed.) The Five Factor Model of Personality: Theoretical Perspectives. (pp. 163-179). New York: Guilford.

Hu, L. T., \& Bentler, P. M. (1999). Cutoff criteria for fit indexes in covariance structure analysis: Conventional criteria versus new alternatives. Structural Equation Modeling: A Multidisciplinary Journal, 6, 1-55. 


\section{http://dx.doi.org/10.1080/10705519909540118}

*Hughes, D. J., Rowe, M., Batey, M., \& Lee, A. (2012). A tale of two sites: Twitter vs. Facebook and the personality predictors of social media usage. Computers in Human Behavior, 28, 561-569. http://dx.doi.org/10.1016/j.chb.2011.11.001

*Ivcevic, Z., \& Ambady, N. (2013). Face to (face) book: the two faces of social behavior? Journal of Personality, 81, $290-301$. http://dx.doi.org/10.1111/j.1467-6494.2012.00804.x

*Jenkins-Guarnieri, M. A., Wright, S. L., \& Johnson, B. D. (2013). The interrelationships among attachment style, personality traits, interpersonal competency, and Facebook use. Psychology of Popular Media Culture, 2, 117-131.

\section{http://dx.doi.org/10.1037/a0030946}

John, O. P., \& Srivastava, S. (1999). The Big Five trait taxonomy: History, measurement, and theoretical perspectives. Handbook of personality: Theory and research, 2(1999), 102-138.

*Kessler, T. T. (2013). Social networking and users: the interaction of personality and motivation for Facebook posting (Unpublished doctoral dissertation), University of Central Florida Orlando, Florida.

*Kim, H., \& Chung, Y. W. (2014). The Use of social networking services and their relationship with the big five personality model and job satisfaction in Korea. Cyberpsychology, Behavior, and Social Networking, 17, 658-663.

http://dx.doi.org/10.1089/cyber.2014.0109 
*Lee, J. E. R., Moore, D. C., Park, E. A., \& Park, S. G. (2012). Who wants to be “friend-rich"? Social compensatory friending on Facebook and the moderating role of public self-consciousness. Computers in Human Behavior, 28, 1036-1043.

http://dx.doi.org/10.1016/j.chb.2012.01.006

*Lee-Won, R. J., Herzog, L., \& Park, S. G. (2015). Hooked on Facebook: The Role of Social Anxiety and Need for Social Assurance in Problematic Use of Facebook. Cyberpsychology, Behavior, and Social Networking, 18, 567-574.

http://dx.doi.org/10.1089/cyber.2015.0002

*Lee-Won, R. J., Shim, M., Joo, Y. K., \& Park, S. G. (2014). Who puts the best "face” forward on Facebook? Positive self-presentation in online social networking and the role of self-consciousness, actual-to-total Friends ratio, and culture.

Computers in Human Behavior, 39, 413-423. http://dx.doi.org/10.1016/j.chb.2014.08.007

Leary, T. (1957). Interpersonal diagnosis of personality: A functional theory and methodology for personality evaluation. New York: Ronald Press.

Liu, D., \& Yang, C. C. (2016). Media Niche of Electronic Communication Channels in Friendship: A Meta-Analysis. Journal of Computer-Mediated Communication. DOI: 10.1111/jcc4.12175

*Lönnqvist, J. E., \& große Deters, F. (2016). Facebook friends, subjective well-being, social support, and personality. Computers in Human Behavior, 55, 113-120. http://dx.doi.org/10.1016/j.chb.2015.09.002 
McCrae, R. R., \& Costa, P. T. (1989). The structure of interpersonal traits: Wiggins's circumplex and the five-factor model. Journal of Personality and Social Psychology, 56, 586-595. http://dx.doi.org/10.1037/0022-3514.56.4.586

McCrae, R. R., \& Costa Jr, P. T. (1997). Personality trait structure as a human universal. American Psychologist, 52, 509-516. http://dx.doi.org/10.1037/0003-066x.52.5.509

*Mishra, V., Niblock, K., \& Shansky, C. (2014). Personality and Facebook Usage as Predictors of Student Participation in Course Blogs. Psychological Studies, 59, 401-407. http://dx.doi.org/10.1037/e537492014-001

*Moore, K., \& McElroy, J. C. (2012). The influence of personality on Facebook usage, wall postings, and regret. Computers in Human Behavior, 28, 267-274. http://dx.doi.org/10.1016/j.chb.2011.09.009

*O'Hanlon, L. (2013). Who's who? What's not to like? Study of personality type, self-esteem, stress and anxiety in relation to Facebook (Unpublished bachelor thesis), Dublin Business School, Dublin, Ireland. Retrieved from http://esource.dbs.ie/bitstream/handle/10788/1635/ba_ohagan_1_2013.pdf?sequence=1

*O'Hanlon, L. (2014). Facebook use and its relationship with personality traits, self-esteem, and internet self-efficacy among college students (Unpublished bachelor thesis), Dublin Business School, Dublin, Ireland. Retrieved from http://esource.dbs.ie/bitstream/handle/10788/2149/ba_ohanlon_1_2014.pdf?sequence=1

*Pettijohn, T. F., LaPiene, K. E., \& Horting, A. L. (2012). Relationships between Facebook intensity, friendship contingent self-esteem, 
and personality in US college students. Cyberpsychology: Journal of Psychosocial Research on Cyberspace, 6, 1-7.

http://dx.doi.org/10.5817/cp2012-1-2

Paulhus, D. L., Robins, R. W., Trzesniewski, K. H., \& Tracy, J. L. (2004). Two replicable suppressor situations in personality research. Multivariate Behavioral Research, 39, 303-328. http://dx.doi.org/10.1207/s15327906mbr3902_7

*Ryan, T., \& Xenos, S. (2011). Who uses Facebook? An investigation into the relationship between the Big Five, shyness, narcissism, loneliness, and Facebook usage. Computers in Human Behavior, 27, 1658-1664. http://dx.doi.org/10.1016/j.chb.2011.02.004

Rizvi, A. Z. (2016). Personality, Social Anxiety and Excessive Use of Facebook. International Journal of Psychology and Behavioral Sciences, 6, 119-127. Doi:10.5923/j.ijpbs.20160603.04

*Simoncic, T. E., Kuhlman, K. R., Vargas, I., Houchins, S., \& Lopez-Duran, N. L. (2014). Facebook use and depressive symptomatology: Investigating the role of neuroticism and extraversion in youth. Computers in Human Behavior, 40, 1-5. http://dx.doi.org/10.1016/j.chb.2014.07.039

*Sin, S. C. J., \& Kim, K. S. (2013). International students' everyday life information seeking: The informational value of social networking sites. Library \& Information Science Research, 35, 107-116. http://dx.doi.org/10.1016/j.lisr.2012.11.006

*Sorokowska, A., Oleszkiewicz, A., Frackowiak, T., Pisanski, K., Chmiel, A., \& Sorokowski, P. (2016). Selfies and personality: Who posts self-portrait photographs? Personality and Individual Differences, 90, 119-123. 


\section{http://dx.doi.org/10.1016/j.paid.2015.10.037}

Suh, E., Diener, E., Oishi, S., \& Triandis, H. C. (1998). The shifting basis of life satisfaction judgments across cultures: Emotions versus norms. Journal of Personality and Social Psychology, 74, 482-493. http://dx.doi.org/10.1037/0022-3514.74.2.482

*Suresh, K. (2013). Social media participatory cultures-a study of the dynamics between user personality and Facebook use. International Journal of Current Research, 5, 925-930.

*Utz, S., Tanis, M., \& Vermeulen, I. (2012). It is all about being popular: The effects of need for popularity on social network site use. Cyberpsychology, Behavior, and Social Networking, 15, 37-42. http://dx.doi.org/10.1089/cyber.2010.0651

Van der Linden, D., te Nijenhuis, J., \& Bakker, A. B. (2010). The general factor of personality: A meta-analysis of Big Five inter-correlations and a criterion-related validity study. Journal of Research in Personality, 44(3), 315-327. http://dx.doi.org/10.1016/j.jrp.2010.03.003

Viswesvaran, C., \& Ones, D. S. (1995). Theory testing: Combining psychometric meta-analysis and structural equations modeling. Personnel Psychology, 48, 865-885.doi: 10.1111/j.1744-6570.1995.tb01784.x

*Wang, C. Y., \& Ching, G. S. (2013). A study on the relationship of Facebook and EFL learners' personality. International Journal of Research Studies in Educational Technology, 2, 3-18. http://dx.doi.org/10.5861/ijrset.2013.434

*Wang, J. L., Jackson, L. A., Zhang, D. J., \& Su, Z. Q. (2012). The relationships among the Big Five Personality factors, self-esteem, 
narcissism, and sensation-seeking to Chinese University students' uses of social networking sites (SNSs). Computers in Human Behavior, 28, 2313-2319. http://dx.doi.org/10.1016/j.chb.2012.07.001

*Wang, S. S. (2013). "I share, therefore I am": personality traits, life satisfaction, and Facebook check-ins. Cyberpsychology, Behavior, and Social Networking, 16, 870-877. http://dx.doi.org/10.1089/cyber.2012.0395

Wiggins, J. S. (1979). A psychological taxonomy of trait-descriptive terms: The interpersonal domain. Journal of Personality and Social Psychology,37, 395-412. http://dx.doi.org/10.1037/0022-3514.37.3.395

*Yao, Z. (2015). The Relationship between Personality and Facebook Usage. Available from http://120.52.72.42/vault.hanover.edu/c3pr90ntcsf0/ altermattw/courses/344/papers/2015/Yao.pdf

*Yesil, M. M. (2014). The relationship between Facebook use and personality traits of university students. International Journal of Academic Research, 6, 75-80. http://dx.doi.org/10.7813/2075-4124.2014/6-2/b.12

*Zabawska, M. (2013). Like it or not. The relationship between personality traits, narcissism, self-esteem, self-monitoring and Facebook usage. (Unpublished bachelor thesis), Dublin Business School, Dublin, Ireland. Retrieved from http://esource.dbs.ie/bitstream/handle/10788/1652/ba_zabawska_m_2013.pdf?sequence=1 